\title{
Promoting creativity and innovation in biotechnology
}

\author{
David J. Adams ${ }^{1,2}$, Lee J. Beniston ${ }^{2} \&$ Peter R.N. Childs ${ }^{3}$ \\ ${ }^{1}$ UK Centre for Bioscience, Higher Education Academy, University of Leeds, Leeds, LS2 9JT; UK \\ ${ }^{2}$ Faculty of Biological Sciences, University of Leeds, Leeds, LS2 9JT; UK \\ ${ }^{3}$ Department of Mechanical Engineering, Imperial College London, South Kensington, London SW7 2AZ, UK
}

Innovative biotechnologists are expected to make major contributions as we emerge from the current period of economic uncertainty. This however is against the backdrop of the vast majority of university students as well as employees in the bioscience industries having received little or no training in techniques that could greatly enhance their creative and innovative potential. Here, we present a range of approaches and strategies designed to promote creativity in bioscientists working in academic and industrial environments.

Recently we sought to establish the extent of creativity training for bioscientists in UK higher education and industry. In academia, of 25 bioscience faculties consulted only four indicated they provide some form of training in creativity methods, but that this generally involved only a minority of students. This no doubt reflects the continuing, massive expansion of the biosciences: students are expected to absorb more and more information and this leaves little opportunity for the development of generative skills. As a result they might feel ill-equipped, for example, for demanding final year projects during which they are expected to demonstrate and adopt creative approaches to problem solving.

A similar situation pertains in industry. When five large UK bioscience companies were consulted about their approach to promotion of creativity in employees, most proved reluctant to divulge information about training programmes, although some indicated that employees receive instruction on creativity methods during management and leadership courses. However, it would appear that none of these organizations provides training programmes designed to promote creativity in the context of bioscience and of the 20 small or medium sized enterprises included in our survey none was able to confirm provision of any form of training in creativity techniques. It therefore seems inevitable that industrial bioscientists will continue to develop ideas that are based only on existing or similar products and this clearly limits the generative power of the individuals involved and the business activity that might arise as a result of this potential.

Much can be done to help bioscientists working in academic or industrial settings to develop their full creative potential. This can be achieved, in part, by identifying working environments and situations that promote the exchange of ideas, and identification of unexpected connections, by colleagues from differing disciplines and perspectives [1]. Individuals might also need help to become aware of their own creative potential. In our experience, students frequently lack the confidence to be creative and need to be reassured, for example, that the person teaching them is often only one step ahead in terms of knowledge and understanding, that the information imparted during lectures is not necessarily written on tablets of stone and that, from 
time to time, it is healthy to question received wisdom and challenge assumptions. It will also help if we can encourage the curiosity about the natural world that probably led bioscientists to study biology in the first place. Indeed it is what we do not know that provides the impetus for research and the resulting exploration of the domain that results in new developments. Individuals who are open-minded, curious and ask lots of questions will be much more inclined to welcome and pursue the unusual result or observation in a research laboratory, or other setting, that might lead to a creative solution to an apparently intractable problem. Further, students and industrialists will benefit greatly from interaction with a wide range of approaches and techniques that may be used to promote creativity in bioscientists working as individuals or in teams.

In a group setting, ideas and suggestions from the more dominant and extrovert participants often rapidly prevail. This may mean that the considerable creative potential of the less forthcoming group members remains untapped and unexploited. Clearly this scenario is extremely wasteful, but can be avoided by encouraging each participant to interact with techniques designed to promote his or her creativity prior to formal group sessions [2]. One approach, that should be attractive to bioscientists, is the identification of an analogy between a problem and a natural phenomenon. Such a 'bioinspiration' approach has resulted in a wide range of innovations and creative solutions to problems ranging from Velcro fasteners to Lotusan (self-cleaning paint) to Pringles Crisps (that occupy little space and remain intact during transit). Literally, hundreds of techniques have been developed, by a very wide range of practitioners, in order to promote creativity in individuals working either alone or in teams [www.mycoted.com]. Students, academics, employers and employees should investigate these as certain approaches will work better for some individuals and situations than for others.

While creativity can and should be encouraged at the individual level it must be stressed that well-managed, interactive group sessions encourage participants to make connections, and identify, share, develop and exploit ideas. A number of key approaches that might be used with groups of participants and that we consider of particular benefit are summarized in Text box 1. These include: stimulatory techniques such as 'brainstorming' and 'checklists'; 'lateral thinking' and 'mind mapping' that promote alternative thinking and the identification of unexpected connections, respectively; the 'six hats' process that promotes parallel, creative thinking in groups; 'morphological analysis', a matrix-based approach to problemsolving; and the more elaborate 'Synectics' and 'TRIZ' techniques that are likely to require expert facilitation. A word of caution regarding brainstorming: while traditional brainstorming sessions, such as those that involve the recording of ideas on flipcharts by a facilitator, can provide certain benefits to organisations (for example, the effective setting of an agenda at the start of a meeting) there is clear and consistent evidence that such approaches are among the least effective ways of generating robust innovative ideas [3]. During these traditional sessions, extroverts often prevail leaving other participants feeling intimidated; as a result, both the group dynamic and collaborative contributions may be suppressed. The problems associated with flipchart brainstorming can, however, be reduced markedly using more carefully structured approaches that encourage simulataneous collective thinking such as 'Grid' or 'Post-it Notes' brainstorming,(Text box 1). Recently, electronic approaches to brainstorming, which allow simultaneous and anonymous contributions, have proved most effective in promoting participation and reducing any anxiety experienced by 
group members, such as the 'Fridge Magnet' approach at www.fbs.leeds.ac.uk/creativity.

Any organisation wishing to encourage idea generation is clearly faced with a wide range of potential creative methods. However, the effectiveness of applying such creative techniques has rarely been evaluated in a controlled, structured manner. This drawback has been addressed in a framework for creative problem solving and generative activity, the 'Create Process', which arose from an EU funded programme, was developed by industrial and academic collaborators and was part of a project that aimed to incorporate 'live' industrial case studies [see: 'Create Project' at www.diegm.uniud.it/create]. The Create Process comprises the following phases: predisposition, external mapping, internal mapping, idea generation and evaluation; and can be implemented using a series of approaches readily recognisable and familiar to the practicing professional (Text box 2). The entire approach has been aligned in order to correlate with Bessant's observations [4], which demonstrated the importance of cumulative incremental innovation. Importantly, the Create Process elements are compatible with the key factors considered to be vital for the release of creative energies in employees, such as goal clarity, freedom to develop diverse ideas, resource definition and provision, and encouragement [5].

The history of mature markets reveals the importance of innovative approaches for maintaining competitive positions [6] and creativity is clearly a key criterion for the effective operation of businesses and the individuals who contribute to them. The bioscience sector, with its implicit understanding of the evolution and survival of ideas, needs to implement strategies to ensure that its personnel are appropriately equipped in order to be able to make and to sustain valuable and novel contributions. This process should start with the promotion of creativity in bioscience students, who would benefit greatly from a more consistent approach across all Higher Education Institutions aided, for example, by the inclusion of 'creativity' and 'innovation' as essential skills for biologists in any future subject benchmark statements for the Biosciences. The policy can then be sustained through a general increase in creativity training with the adoption of frameworks such as the Create Process in industrial and other settings. It is to be hoped that a strategy of this nature will help overcome the bottlenecks that confront the biotechnologist in areas of great current significance such as bioengineering, drug discovery and stem cell research/therapy. 


\section{References}

1. Neumann, C.J. (2007) Fostering creativity. A model for developing a culture of collective creativity in science. EMBO Reports 8, 202-206.

2. Adams, D.J. \& Grimshaw, P. (2008) Creativity and innovation in the biosciences. In Enterprise for Life Scientists: Developing Innovation and Entrepreneurship in the Biosciences (Adams, D.J. and Sparrow, J.C., eds), pp. 25-51, Scion.

3. Furnham, A. (2000) The brainstorming myth. Business Strategy Review 11, 21-28.

4. Bessant, J. (1992) Big bang or continuous evolution: why incremental innovation is gaining attention in successful organisations. Journal of Creativity and Innovation Management 1, 59-63.

5. Burnside, R.M. (1988) Encouragement as the elixir of innovation. Issues and Observations 8, 1-6.

6. Cox, G. (2005) Review of creativity in business: building on the UK's strengths. HM Treasury, UK.

7. Osborn, A.F. (1963) Applied Imagination: Principles and Procedures of Creative Problem Solving. $3^{\text {rd }}$ edition, Scribner's.

8. Michalko, M. (2001) Cracking Creativity, Ten Speed Press.

9. Zwicky, F. (1969) Discovery, Invention, Research through the Morphological Analysis, Macmillan.

10. Childs, P.R.N. (2004) Mechanical Design, Butterworth Heinemann, Elsevier.

11. De Bono, E. (1970) Lateral Thinking: Creativity Step by Step, Harper \& Row.

12. De Bono, E. (1999) Six Thinking Hats, Penguin.

13. Buzan, T. \& Buzan, B. (2006) The Mind Map Book, Revised edition, BBC Active.

14. Gordon, W.J.J. (1961) Synectics: the Development of Creative Capacity, pp. 3-7 and 33-56, Harper \& Row.

15. Altshuller, G.S. (1984) Creativity as an Exact Science - the Theory of the Solution of Inventive Problems, Gordon \& Breach. 


\section{Box 1. Some techniques for the promotion of creativity}

\section{Effective brainstorming techniques e.g. 'Post-it' or 'Grid' [8]}

In 'Post-it' participants record ideas on Post-it notes and these are collected, reviewed and analysed. In 'Grid' a participant records some solutions to a problem on a grid that is completed by other members of the group. These techniques encourage simultaneous generative activity but some participants may remain reluctant to share an idea for fear of ridicule or loss of ownership. The latter concern may be addressed by implementation of IPR protocol.

\section{Checklists [7]}

A series of brief questions and/or statements is used to stimulate creativity when it's proving difficult to think in new and original ways. Can be used for idea generation and evaluation but is a systematic method that may not appeal to all personality traits.

\section{Lateral thinking [11]}

Solutions are proposed by looking at a problem using random associations, provocation, challenging current solutions and divergence. Requires both curiosity and confidence and encourages a wide range of alternative solutions. Robust evaluation of ideas is necessary to identify worthwhile concepts to develop.

\section{Mind mapping [13]}

Connections between associated pieces of information are emphasised by clustering the information on a visual map: this can stimulate creativity. Some people are hesitant to reveal a perceived weakness in sketching but this can be overcome with practice.

\section{Six hats [12]}

Parallel thinking process in which team members wear coloured hats representing data, creativity, positivity, feelings, criticism and control. The approach can minimise conflict, encourage participation and consideration of a problem from a wide range of perspectives. However, some participants may be hesitant to take this broad-minded approach.

\section{Morphological analysis $[\mathbf{9 , 1 0}]$}

A matrix-based technique in which a problem is broken-down into component parts and a range of approaches/solutions suggested for each of these elements. Encourages combinations of features and solutions that otherwise might not have been considered but large quantity of combinations generated means that good ideas can be overlooked.

Weighting criteria should therefore be used to guide selection of solutions.

\section{Synectics [14]}

Exploits our capacity to connect apparently irrelevant elements to spark new ideas and solutions. The approach helps participants break existing mind sets and internalize abstract concepts but is time-consuming, requiring practice and expert facilitation.

\section{TRIZ [15]}

Russian acronym for the Theory of Inventive Problem Solving. Provides a framework and toolbox for systematic, inventive problem solving but is sometimes viewed as complicated and difficult to use; this can be overcome by sustained use and practice. 


\section{Box 2: The Create Process}

\section{Predisposition.}

Provides a work environment that: allows expression of individual creativity; promotes a creative culture; clearly identifies leaders and facilitators. Can be achieved by training in creativity and innovation and change management processes.

\section{External Mapping}

Analyse the environment out side the organization to identify e.g.: new needs and opportunities; talented individuals; the economic implications of entering a market. A questionnaire survey and analysis, known as Attribute Value Chain, can be used to produce a mental map to stimulate the next phase of idea generation.

\section{Internal Mapping}

Analyse the organization concerned using, for example, SWOT analysis to identify Strengths,

Weaknesses, Opportunities, Threats.

\section{Idea Generation}

Promote creativity at individual and group level. See Text box 1 and main text.

\section{Evaluation}

Select the best ideas to emerge using internal assessment criteria. De Bono's Six Thinking Hats (Text box 1) can also be used for idea evaluation.

(see: www.diegm.uniud.it/create) 